\title{
CORRIGENDUM
}

\section{Does sexism affect Thai women's psychological and behavioral responses? The stereotype threat-buffering effect of mindfulness - CORRIGENDUM}

Prapimpa Jarunratanakul \& Kamonkan Jinchang

https://doi.org/10.1017/prp.2018.10, Published by Cambridge University Press, 19 June 2018

Keywords: sexism, stereotype threat, identity separation, career aspirations, mindfulness, corrigendum

The authors apologize for error in the abstract (Jarunratanakul \& Jinchang 2018). The correct version is given below

Abstract: Few studies have examined the linkage of perceived sexism with stereotype threat and psychological responses in a work setting and moderators that may buffer this negative effect. In Study 1, the relationships among perceived sexism, stereotype threat, and psychological responses were explored among Thai female employees $(\mathrm{N}=296)$ in a self-report questionnaire study. The aim of Study 2 was to investigate whether mindfulness can mitigate the disastrous effect of sexism on performance, using an experimental design. In Study 1, the structural equation modeling (SEM) results observed a partial mediation effect of stereotype threat for the sexism-disidentification relationship, and sexism only had a significant direct effect on career aspirations. In Study 2, the interaction effect of sexism $\times$ mindfulness on the female participants' reasoning test scores was significant, indicating that those with mindfulness practice performed better when being exposed to sexist behaviours. The current research provides additional information on understanding the impact of sexism on disengagement and decreased career aspirations among Thai female employees in male-dominated industries. In practice, Thai organisations should be concerned about the impact of sexism on disengagement and the protective factor of performance decrements by encouraging anti-sexist norms and fostering the cultivation of mindfulness through practice.

\section{Reference}

Jarunratanakul, P., \& Jinchang, K. (2018). Does sexism affect Thai women's psychological and behavioral responses? The stereotype threat-buffering effect of mindfulness. Journal of Pacific Rim Psychology, 12, E16. doi: 10.1017/prp.2018.10 